\title{
Failure Analysis of Polyethylene Products Using Microscopy
}

\author{
Ann Johnson, Praveenkumar Boopalachandran, Eddy Garcia-Meitin ${ }^{1}$ and Siddharth Ram Athreya ${ }^{2}$ \\ 1. The Dow Chemical Company, Analytical Sciences Microscopy, Lake Jackson, USA. \\ 2. The Dow Chemical Company, Packaging and Specialty Plastics, Lake Jackson, USA.
}

Failure analysis of fabricated products is approached using analytical techniques such as light and electron microscopies coupled with compositional microanalysis. The advantage of looking through the lens of a microscope allows careful surveying of the failure and helps identify regions for further investigation [1]. These techniques can be used to troubleshoot failure in applications ranging from flexible food packaging films to larger parts such as composites and pipe. Understanding the root cause of failure is a key variable in resolving production issues and is leveraged towards product development and design at Dow [2]. This paper highlights how light and scanning electron microscopies, coupled with hot stage and infrared spectroscopy, were used to identify the root cause of failure in polyethylene tubing.

Defects in fabricated products can result from a variety of sources such as poor component mixing or contamination that occurs during manufacturing or fabrication. Fabrication defects can include resin gels, foreign contaminants or poor pigment dispersion that can compromise product performance. Two case studies will be discussed to demonstrate how microscopy and microanalysis were leveraged to systematically examine and identify the root cause of failure in polyethylene tubing.

In the first example, a black tubing (A) attached to a connector fitting failed service. Light microscopy was used to survey the tubing and determine that a crack occurred at the fitting (Figures 1a-b). A razor blade was used to isolate the failed joint by cutting $2 \mathrm{~mm}$ thick slices perpendicular to the longitudinal tubing crack near the crack arrest. Low magnification optical analysis of razor cut cross sections provided evidence that crack initiation occurred internally near the interior tubing surface. This finding was evident by the remaining polymer ligaments holding the tubing walls together (Figure 1c). To further investigate the crack arrest, a cryogenic microtome with a diamond knife was used to collect $5 \mu \mathrm{m}$ thick cross sections for transmitted light microscopy (Figures 1d-f). Cross sections were examined under transmitted bright-field illumination on a compound microscope. Examination of the crack clearly showed that swirl lines associated with poor carbon black pigment dispersion had initiated internal cracks which propagated along pigment rich interfacial boundaries (Figure 1f). This work showed that poor carbon black pigment dispersion associated with "salt and pepper" dry blending of pigment produced poor pigment dispersion resulting in weak interfacial boundaries that led to connector failures. Melt blending of pigments prior to fabrication can improve dispersion and mitigate inhomogeneity that can influence product performance.

In a second example, a black tubing (B) developed a visible surface defect resembling a gel or inclusion upon production (Figure 2a). Gels are defects that can develop from unmelt polymer, pigments, crosslinking, or foreign contamination during production, handling or fabrication [3]. Others in the industry may also refer to them as inclusions due to the variety of ways that defects form [4]. Analysis of $5 \mu \mathrm{m}$ thick cross sections under transmitted bright field (Figure 2b) and epifluorescence illumination showed a highly brittle material that fluoresced under ultraviolet light (Figure 2c). Further investigation using energy dispersive $\mathrm{x}$-ray (EDX) and infrared micro spectroscopy determined that the gel was organic and 
consistent with oxidized polyethylene. The source of the gel was traced to dirty extrusion equipment which contaminated the tubing during fabrication.

A variety of analytical techniques were used to understand and identify failures associated with black polyethylene tubing. Proper application of these techniques in a systematic approach are key in understanding failure and product development at Dow.

\section{References:}

[1] L Sawyer and D Grubb in "Polymer Microscopy", (Springer Netherlands) p. 12, p. 357.

[2] E Garcia-Meitin et al., SPE ANTEC 2015 Tech Papers, p.2375.

[3] MA Spalding et al., Journal of Plastic Film \& Sheeting 34(3) (2018), p. 300.

[4] A Gel By Any Other Name, https://www.ptonline.com (accessed December 20, 2018).
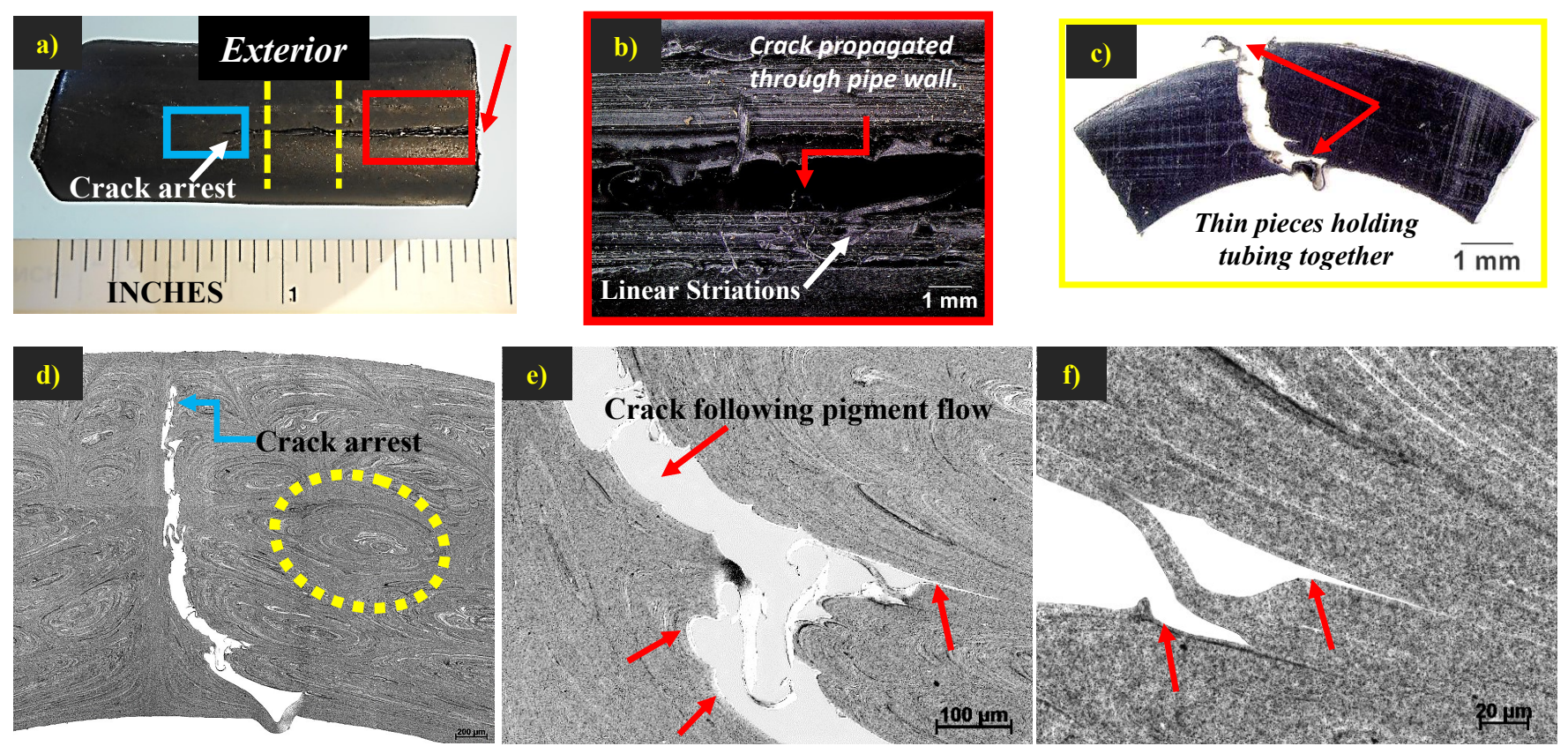

Figure 1. Black tubing (A). a) Tubing with linear crack which formed at fitting, b) exterior top-down view of exterior surface with open crack, c) $2 \mathrm{~mm}$ thick cross section ligaments on interior and exterior surfaces, d) $5 \mu \mathrm{m}$ thick cross sections of crack and arrest, e) magnified view showing crack path following pigment interfacial boundaries along pipe interior, f) higher magnification of image in e.
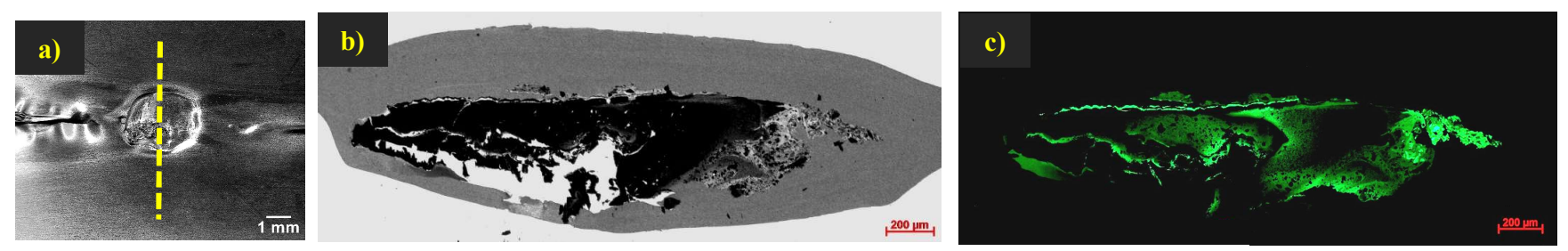

Figure 2. Black tubing (B). a) Top down image of a gel identified from the edge of a blister defect, and b) transmitted bright-field image of $5 \mu \mathrm{m}$ thick cross section taken through the gel, c) epi-fluorescence image of gel showing an intense fluorescence emission consistent with polymer oxidation. 